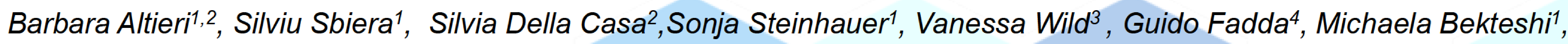
Andreas Rosenwald ${ }^{3}$, Alfredo Pontecorvi ${ }^{2}$, Martin Fassnacht ${ }^{1}$,Bruno Allolio ${ }^{1}$, Cristina L. Ronchi' .

\title{
Inhibitor of apoptosis protein livin/BIRC7 in adrenocortical tumors.
}

\author{
${ }^{1}$ Endocrine and Diabetes Unit, Department of Internal Medicine I, University Hospital of Wuerzburg, Germany. \\ 2 Institute of Medical Pathology, Division of Endocrinology and Metabolic Diseases, Catholic University, Rome, Italy. \\ ${ }^{3}$ Department of Pathology, University Hospital of Wuerzburg, Germany. \\ ${ }^{4}$ Division of Anatomic Pathology and Histology, Catholic University, Rome, Italy
}

INTRODUCTION: Adrenocortical tumors (ACT) comprise frequent benign adenoma (ACA) and rare highly malignant carcinoma (ACC). Livin/ML-IAP/BIRC7 is a member of the inhibitors of apoptosis proteins family, which are involved in cancer development, progression and resistance to chemotherapy in several human malignancies, mostly through the direct inhibition of caspase-3 (Fig.1). Aim of the study was to evaluate the expression of BIRC7/livin and its isoforms livin $\alpha$ and livin $\beta$ in normal and neoplastic adrenal glands.

METHODS: The mRNA expression of BIRC7, its isoforms livin $\alpha$ and livin $\beta$, and caspase-3 was evaluated by quantitative real-time RT-PCR analysis in 84 fresh-frozen tissue samples (34 ACC, $25 \mathrm{ACA}$, and 25 normal adrenal glands=NAG) (Tab.1), including 19 paired samples of tumor and surrounding normal adrenal tissue (13 ACA and 6 ACC). The mean value of the threshold cycle was normalized with $\beta$-actin ( $\Delta$ Ct value). Specific primer were used to amplify 216-bp livin $\alpha$ and 162-bp livin $\beta$. Livin isoforms were then identified by $4 x$ agarose gel in all paired samples.

Additionally, livin protein expression was assessed by western blot analysis (WB) in a subgroup of 15 paired samples (10 ACA and 5 ACC with surrounding normal adrenal tissue). Livin immunostaining ( $\mathrm{HC})$ was evaluated in both cytoplasm and nuclei by $\mathrm{H}$ score in 127 paraffin-embedded tissue sections (67 ACC, 45 ACA, 15 NAG). The antibody used for WB and IHC was livin policlonal antibody (NB100-56145, NovusBio, 1:1000) which recognized both isoforms $\alpha$ and $\beta$. The relationship with several histopathological and clinical data was also evaluated.

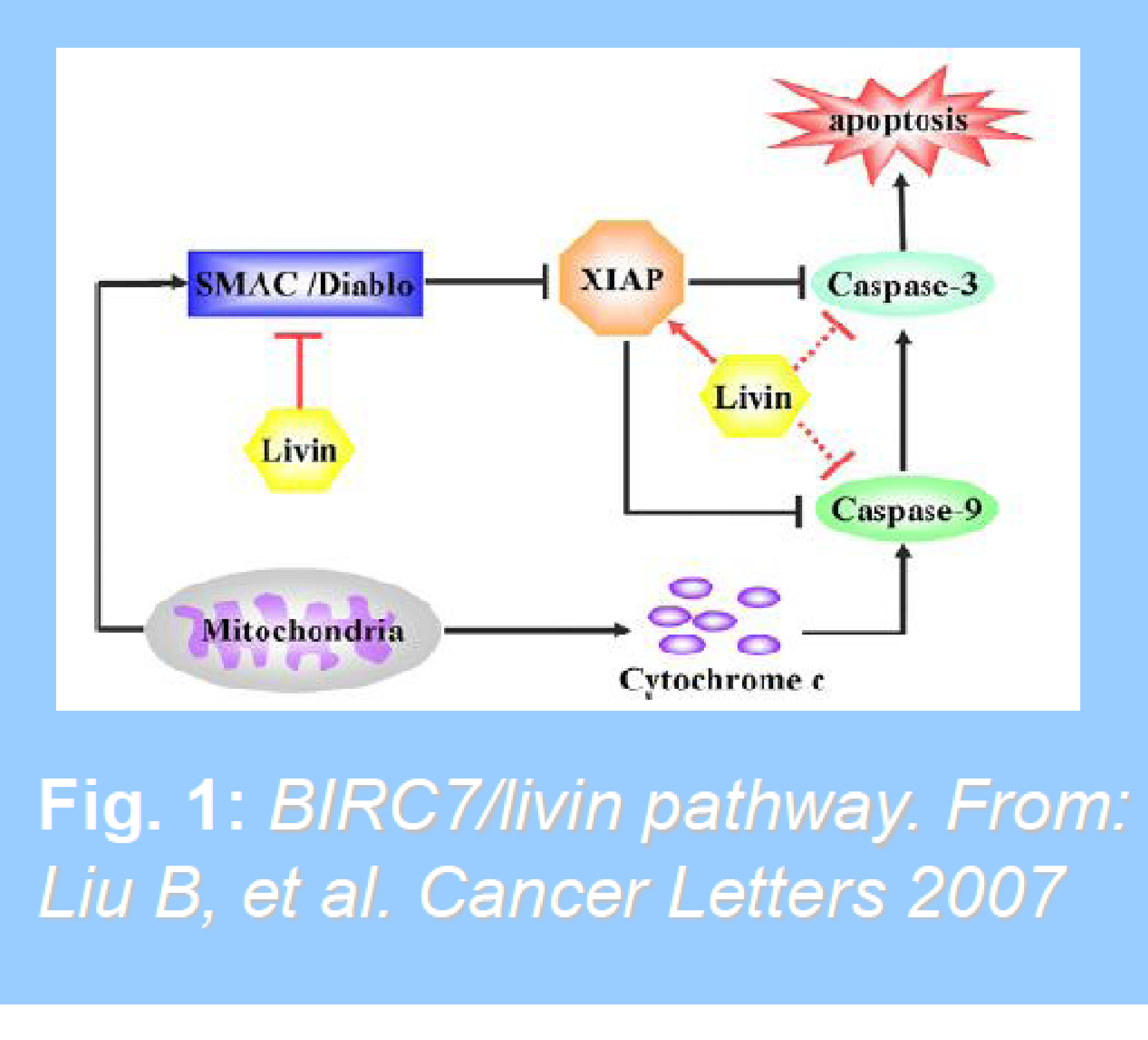

RESULT: relative BIRC7 mRNA expression was similar between ACAs $(0.01 \pm 0.01)$ and NAG $(0.01 \pm 0.02)$, but significantly higher in ACCs $(0.06 \pm 0.12, P<0.005$ vs both ACA and NAG) (Fig.2A), being more expressed in tumors than in surrounding normal tissues in paired samples (Fig.2B). Both isoforms $\alpha$ and $\beta$ were detected in normal and tumor tissues (Fig.3), livin $\beta$ being constantly higher than livin $\alpha(P=0.07$ in ACC; $P=0.10$ in ACA; $P=0.02$ in NA). Comparable results were obtained with WB (Fig.4).

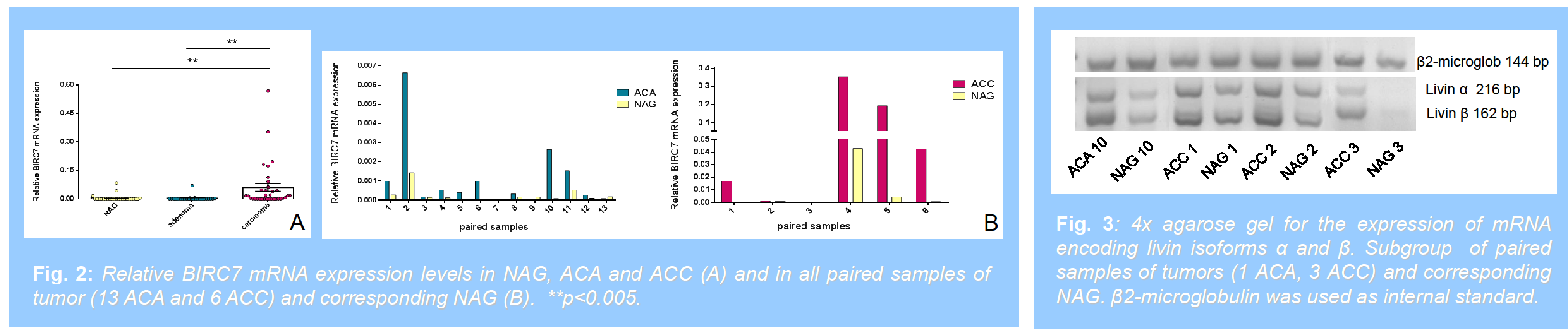

Livin protein expression in the cytoplasm was generally higher in ACC than ACA and NAG (mean $\pm S D$ H-score: $1.72 \pm 0.7$ vs $1.58 \pm 0.5$ vs $1.33 \pm 0.6$ respectively, $P=0.09$ ) (Fig.5A), often being stronger in the tumor than in surrounding normal adrenal tissue (Fig. 5B). At nuclear level, livin protein was highly expressed in both ACAs and ACCs, but almost absent in NAG $(P<0.001$ per trend) (Fig.6). No significant correlation was observed with the histopathological and clinical data, including overall survival.

Caspase-3 mRNA level was higher in ACA than in ACC $(P=0.05)$ and NAG $(P=0.03)$, with a significant inverse correlation with tumor size $(P=0.005, R=0.36)$.
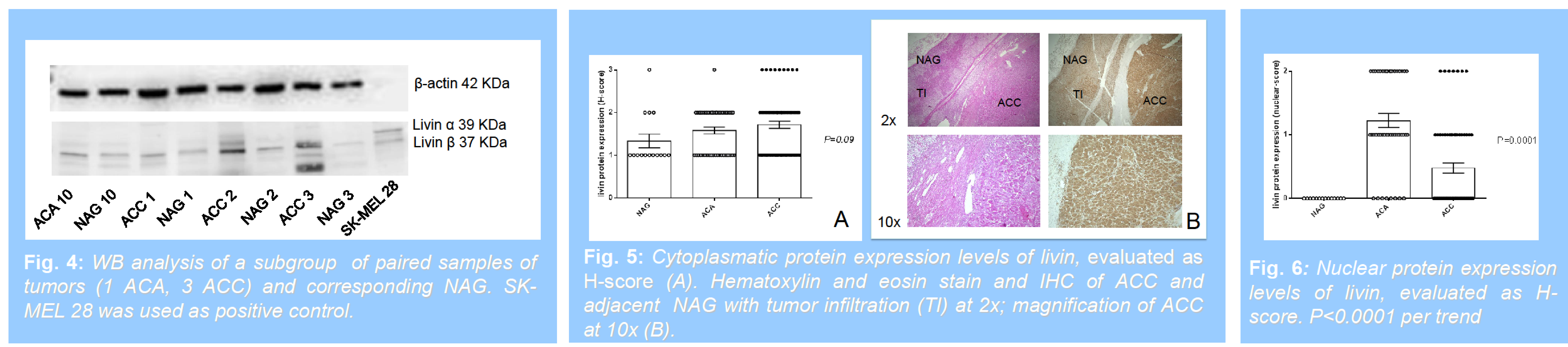

CONCLUSION: Our study demonstrates that BIRC7/livin is specifically over-expressed in ACC, suggesting that it may be involved in adrenocortical tumorigenesis. BIRC7 could represent a novel marker for malignancy and a promising tool for targeted therapy in ACC.

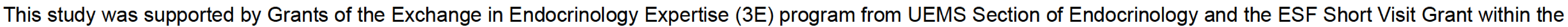
framework of the ESF activity entitled "European Network for the Study of Adrenal Tumors". 